\title{
La roue pleine et ses dérivés
}

The solid wheel and its derivatives

\section{Jean Spruytte}

\section{OpenEdition}

Journals

Édition électronique

URL : https://journals.openedition.org/tc/5015

DOI : $10.4000 /$ tc. 5015

ISSN : 1952-420X

\section{Éditeur}

Éditions de l'EHESS

\section{Édition imprimée}

Date de publication : 30 juin 2010

Pagination : 473-483

ISSN : 0248-6016

\section{Référence électronique}

Jean Spruytte, «La roue pleine et ses dérivés », Techniques \& Culture [En ligne], 54-55 | 2010, mis en ligne le 30 juin 2013, consulté le 29 septembre 2022. URL : http://journals.openedition.org/tc/5015 ; DOI : https://doi.org/10.4000/tc.5015 


\section{LA ROUE PLEINE ET SES DÉRIVÉS}

in Techniques et culture 6, 1985 : 99-110

L'importance des découvertes archéologiques de roues pleines et les différentes représentations de ce type de roue sur les documents figurés de l'Antiquité peuvent permettre de proposer une chronologie technique fondée sur l'étude expérimentale et l'analyse technologique. Cette étude fait apparaître les facteurs matériels qui ont conditionné l'évolution technique de la fabrication des roues pleines (car ces contraintes, d'ordre pratique, sont invariables à travers l'espace et le temps et peuvent être identifiées expérimentalement) ; elle permet d'autre part de mettre en évidence, sur des véhicules encore en usage au $\mathrm{xx}^{e}$ siècle, la persistance de deux dérivés de la roue pleine originelle dont la conception technique remonte au début du II $^{\mathrm{e}}$ millénaire avant notre ère.

\section{Considérations préliminaires sur la roue}

On a pu supposer que l'invention de la roue pleine avait pour origine le rouleau, cylindre de bois placé sous une charge pour la déplacer, et dans lequel des disques auraient été découpés transversalement. Cette supposition ne résiste pas à l'analyse, pour différentes raisons.

En chronologie historique, l'utilisation pratique du rouleau n'apparaît en premier lieu que sur les bas-reliefs Assyriens du ix siècle avant J.-C., alors que la roue pleine est attestée à la fois par les découvertes archéologiques et les documents figurés dès le III $^{e}$ millénaire. 


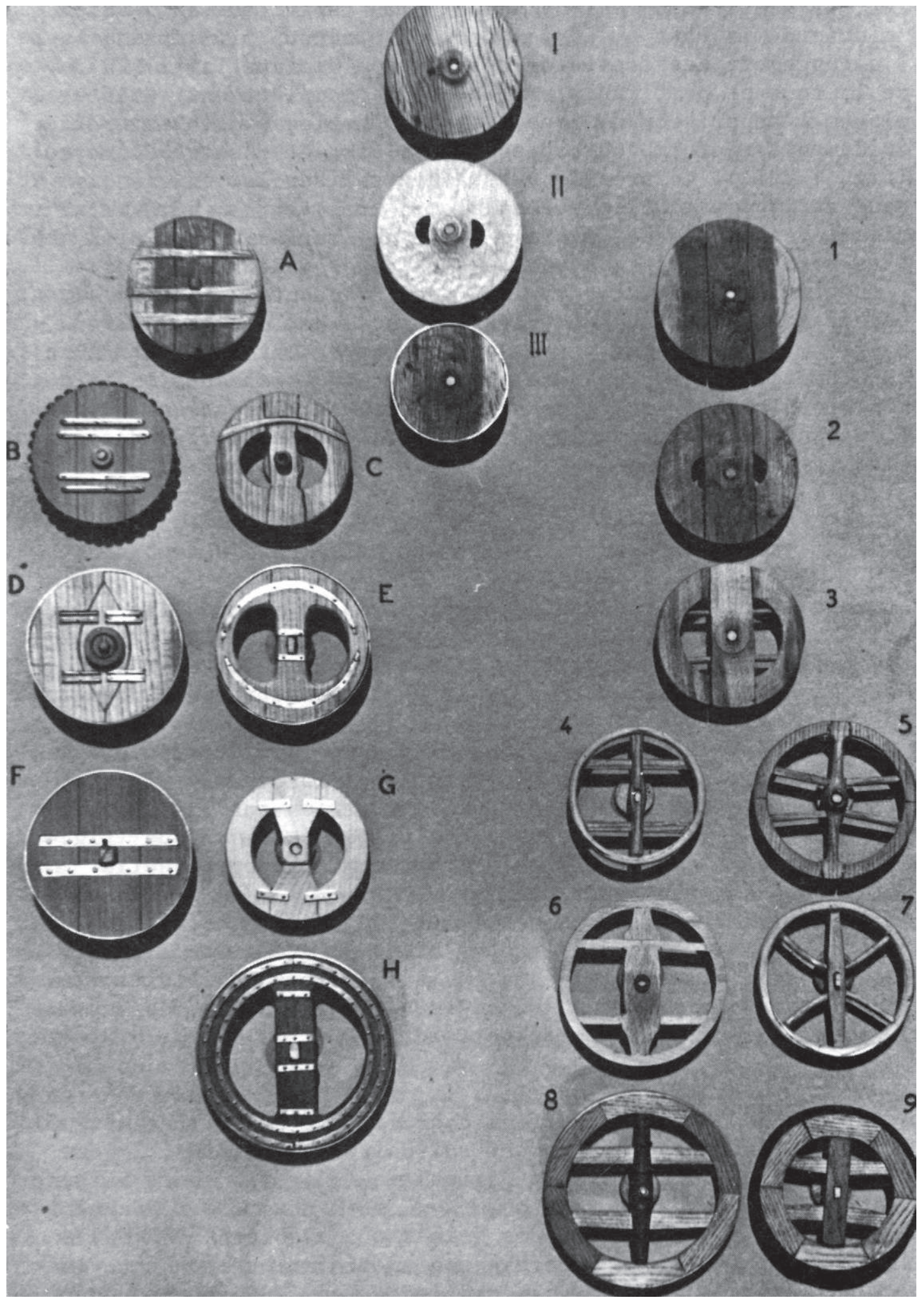

Planche des roues

Maquettes au 1/10 des roues citées et étudiées au justificatif archéologique. (Fig. 1) 


\section{SOURCES DES RECONSTITUTIONS DE ROUES PRÉSENTÉES DANS LA FIGURE 1}

\begin{tabular}{|c|c|}
\hline ROUES & SOURCES \\
\hline I- II - 2 et $C$ & $\begin{array}{l}\text { Van der Waals, J.0., « Neolithic disc wheels in the Netherlands », Palaeohistoria X (1964). } \\
\text { Lucas, A. T., « Prehistoric block-wheels from Doogarymore c.o. Rosconmon, and Timahoeeast, co. Kildare », Journal the } \\
\text { royal society antiquaries, Ireland Lll (1972). }\end{array}$ \\
\hline III et $\mathrm{F}$ & Roues de charrettes basques - Musée basque, Bayonne. \\
\hline A & Ruoff, U., « Die schnurkeramischen rader von Zurich Pressehauss », Archaologisches Korrespondenzblatt VIII (1978). \\
\hline B & Littauer, M.A. et J. Crouwel, « Wheeled vehicles and ridden animals in the ancient near east », Brill(1979). \\
\hline D & Documents figurés - roue sumérienne. \\
\hline $\mathrm{E}$ & Portugal - documentation photographique de M. Gast. \\
\hline G & Balouchistan - Heyerdahl, T., Tigris. Paris : Albin Michel (1979). \\
\hline H. & Espagne -documentation photographique. \\
\hline 1. & Musée historique de la Géorgie. Tibilissi. U.R.S.S. \\
\hline 2. & $\begin{array}{l}\text { Piggott, S., « A tripartite dise wheel from Blair Drummond, Perthshire », Proceedings of the society of antiquaries of } \\
\text { Scotland, vol. XC, session 1956-57. }\end{array}$ \\
\hline 3. & $\begin{array}{l}\text { Atkinson, F. et A. Ward, « A pair of 'cloq !' wheels from northern England », The Yorkshire dialect society, vol. XI Part. } \\
\text { LXIV (1965). }\end{array}$ \\
\hline 4. & Documents figurés grecs, roue des charrettes de transport, ve siècle av. J.C. \\
\hline 5. & Roue de Mercurago - Musée de Turin. \\
\hline 6. & Roue d'Ouroux - Archéodrome de Beaune. \\
\hline 7. & Roue du sarcophage de Vulci. \\
\hline 8. & Littauer, M.A. et J. Crouwel, « The origin and diffusion of the cross bar wheel », Antiquity LI (1977). \\
\hline 9. & Mandchourie - Document photographique Aulagnon « L'Homme », Collection Larousse. \\
\hline
\end{tabular}


Mise hors service d'une roue sans renflement central par usure du moyeu.

(Fig. 2)
La roue pleine monobloc, débitée transversalement au fût de l'arbre (en « bois de travers » en termes de métier) n’a jamais été attestée à ce jour par des découvertes de fouilles.

Enfin, il existe une différence technique fondamentale entre le rouleau et la roue : le rouleau supporte la charge par son diamètre et la fait avancer par le développement de sa circonférence ; le point de support, constamment variable, se déplace donc vers l'arrière lorsque la charge avance. La roue, elle, supporte la charge par son rayon; le point de support reste constamment fixe et se déplace avec la charge.

Il en découle que dans l'élaboration de la roue, l'invention capitale a été le moyeu, qui a permis de ramener le point de support au rayon.

La roue se définit comme un organe circulaire tournant autour de son centre et toutes les roues, ainsi que toutes les applications qui en dérivent (engrenages, turbines, poulies, volants, etc.), répondent à cette définition.

Cette invention primordiale a été la base de presque toute l'évolution technique et matérielle ayant abouti à la civilisation actuelle (l'ordinateur lui-même en est tributaire). Cela justifie la recherche fondamentale sur ses origines et sur la chronologie de son développement technique et technologique.

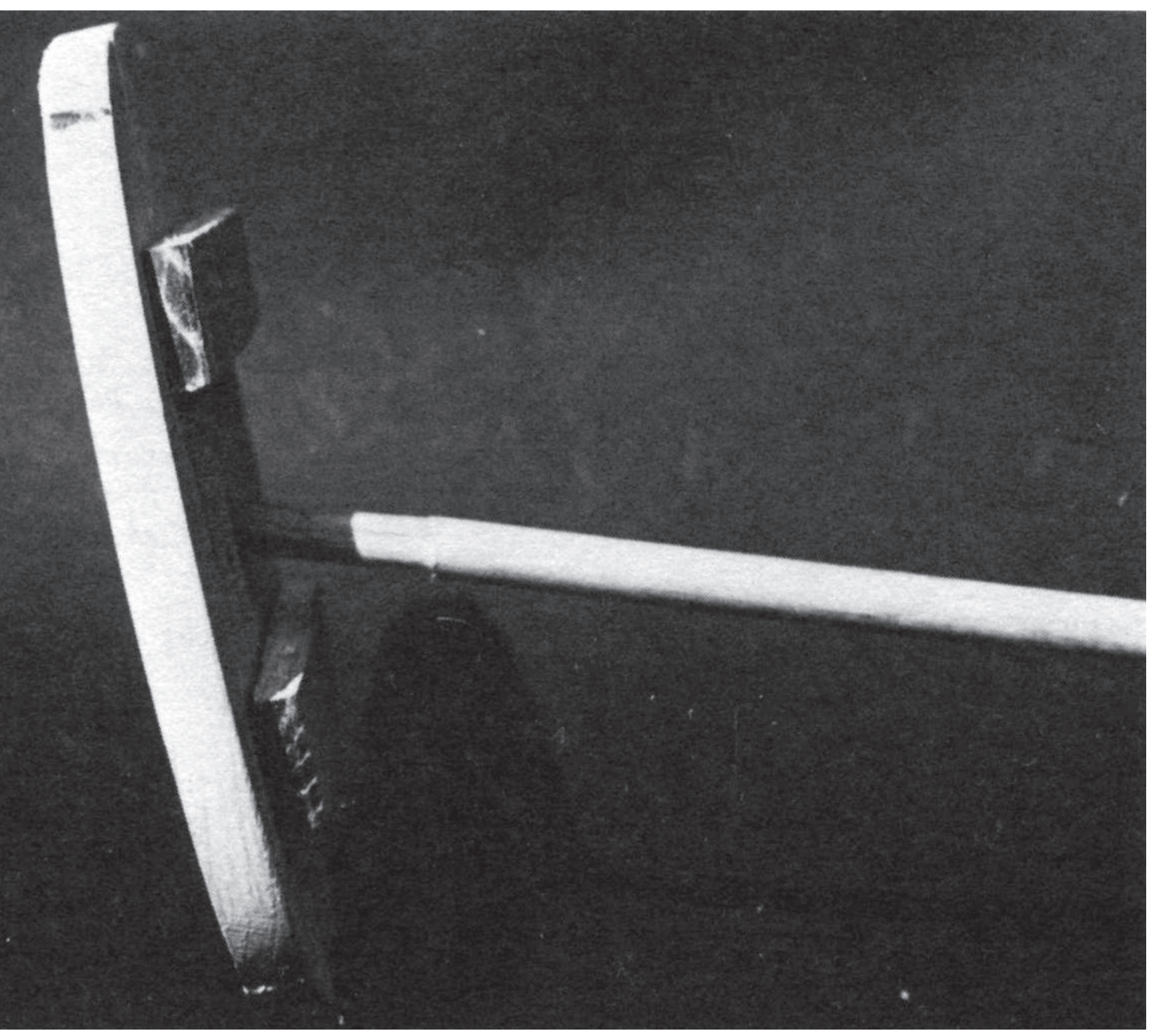




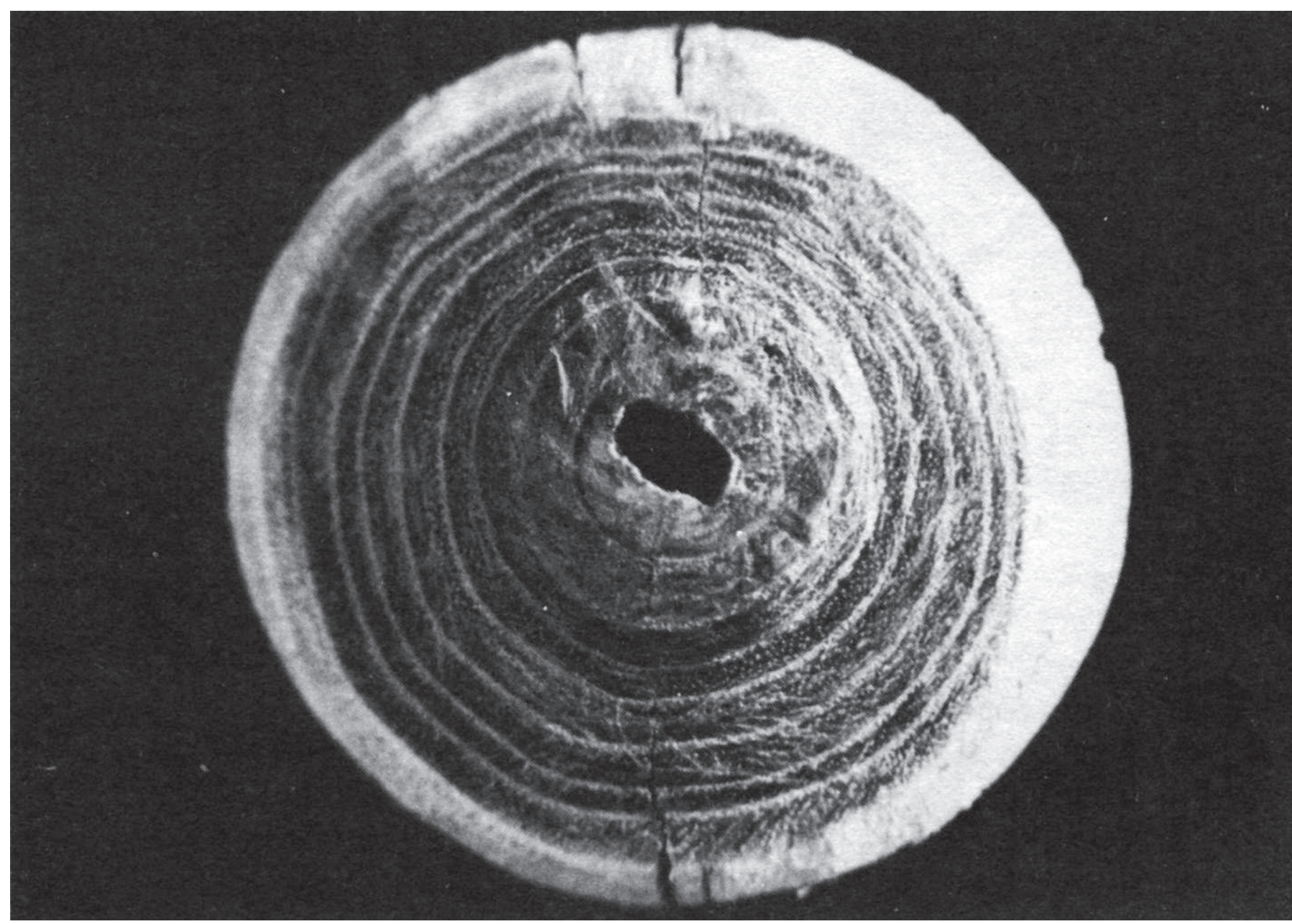

\section{La roue pleine monobloc : contraintes de fabrication et défectuosités}

Fentes par dessiccation sur la partie amincie d'une roue débitée en " bois de travers ».

(Fig.3)

Dès son apparition, la roue pleine monobloc se présente comme un disque renflé en son centre et aminci sur son pourtour (fig. 1). Cette forme est imposée par l'obligation d'avoir suffisamment d'épaisseur au centre pour obtenir un moyeu assez long permettant une bonne stabilité de la roue.

Si cette condition n'était pas respectée, le jeu entre le moyeu et la fusée de l'essieu deviendrait rapidement excessif, par suite de l'usure et de la dessiccation des bois, et la roue oscillerait jusqu'à l'écrasement ou la rupture de la fusée (fig. 2).

Les roues pleines monobloc découvertes à ce jour sont toutes fabriquées en bois « de fil », c'est-à-dire dans une forte planche obtenue dans la longueur d'un fût d'arbre au maximum de son diamètre et débitée soit par sciage, soit par fendage (débit en « merrain » à l'aide de coins). Il est d'ailleurs exclu qu'une roue pleine monobloc puisse être débitée perpendiculairement à la longueur du fût, car un disque aminci en « bois de travers » se fend à la dessiccation (fig. 3) et d'autre part, parce que le bois coupé en contrefil n'offre aucune résistance aux chocs latéraux.

La roue pleine monobloc fabriquée en bois de fil est néanmoins soumise à plusieurs contraintes. Tout d'abord, son diamètre ne peut être supérieur à celui de l'arbre dont 
elle provient et, lors de la dessiccation du bois, des fentes, dans le sens du fil, apparaissent généralement sur les deux côtés opposés de la périphérie correspondant aux extrémités de la planche initiale (appelée « le bois de bout » en termes de métier). En effet, la dessiccation est nettement plus rapide sur la partie amincie de la roue qu'en son centre, beaucoup plus épais ; et l'allongement des fentes de dessiccation diminue la solidité de la roue et peut finir par la fendre complètement. Cela explique l'apparition de roue pleines monobloc présentant deux évidements en forme de croissant, de part et d'autre du moyeu (fig. 1 : II). Le procédé vise à neutraliser les effets inégaux de la dessiccation sur un bloc d'épaisseur variable, en créant des espaces d'aération et de dilatation aux points de plus forte résistance à une dessiccation rapide.

Cette amélioration technique dénote incontestablement une connaissance certaine du travail du bois dès le III $^{e}$ millénaire, mais ne résout pas le problème posé par la limitation du diamètre de la roue à celui de l'arbre. Cependant, la roue monobloc initiale se retrouvera aux temps modernes quand un solide bandage de fer empêchera toute détérioration par dessiccation (fig. 1 : III).

\section{La roue pleine tripartite : les deux procédés d’assemblage}

La roue pleine tripartite qui apparaît à la fin du $\mathrm{III}^{\mathrm{e}}$ millénaire constitue la solution technique à ce problème du diamètre : il n'est plus désormais tributaire de celui de l'arbre. Cette importante innovation va de plus permettre une évolution vers deux autres types de roues.

La solidité d'une roue tripartite dépend du procédé d'assemblage de ses trois pièces constitutives; ce type de construction est en effet soumis lors de sa rotation sur le sol à quatre effets de cisaillement successifs par tour de roue (fig. 4), et la force de ces effets est directement proportionnelle au poids du chargement supporté.

Il est remarquable que dès l'origine, deux procédés relevant de technologies différentes aient pu résoudre le problème posé ${ }^{1}$, et cela d'une manière tellement définitive que l'on retrouvera ces mêmes procédés appliqués aux mêmes types de roues encore en usage aujourd'hui.

Lassemblage à goujons (fig. $1: 1$ ) : des goujons pénètrent à force dans les trois parties constitutives de la roue (fig.5) et les maintiennent absolument solidaires ; néanmoins, la dessiccation, plus rapide sur la périphérie (pour les mêmes raisons que dans le cas de la roue monobloc), aura toujours tendance à disjoindre les extrémités des joints d'assemblage (visibles sur la fig. $1: 1$ ) et imposera une amélioration technique qui est à l'origine d'un nouveau modèle de roue, directement dérivé de cette modification.

L'assemblage à éclisses (fig. 1: A) : ce procédé assure une liaison parfaite des trois parties constitutives de la roue, grâce à trois éclisses, naturellement en forme de coins, mais de section trapézoïdale (en queue d'aronde) (fig. 6). L'éclisse centrale verrouille d'autre part une fusée d'essieu carrée (la roue est clavetée sur l'essieu qui lui-même tourne dans des échantignolles sous la caisse du véhicule). Ce type de roue est sujet, comme le précédent et toujours pour les mêmes raisons, aux effets de la dessiccation; et il bénéficiera de deux améliorations différentes : l'une, à l'âge des métaux, conserve la structure initiale, tandis que de l'autre, dérive directement un nouveau modèle de roue. 
Effets de cisaillement sur une roue pleine tripartite lors du roulement.

(Fig.4)

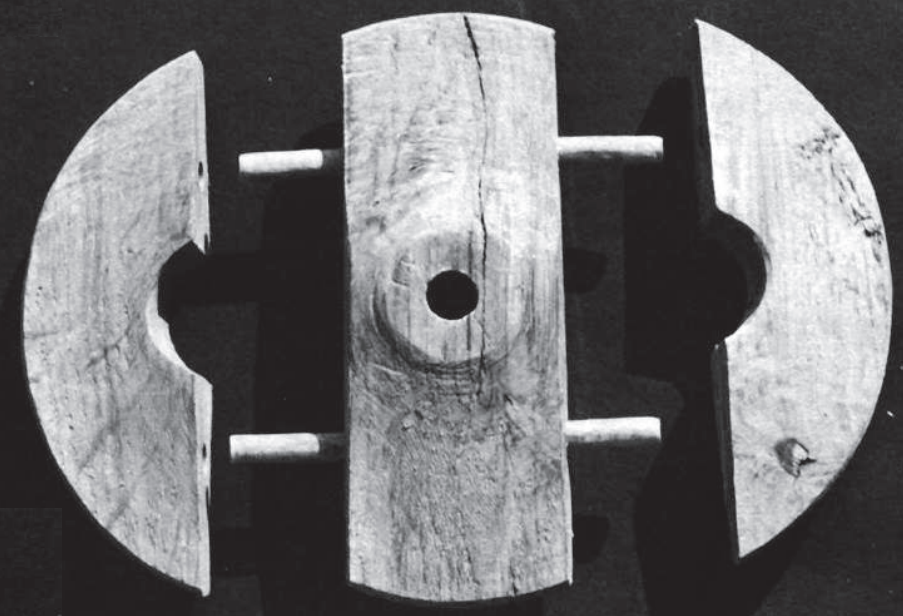

Assemblage à éclisses

(roue A de la planche).

(Fig.6)

Assemblage à éclisses avec modification de structure (roue B de la planche).

(Fig.7)
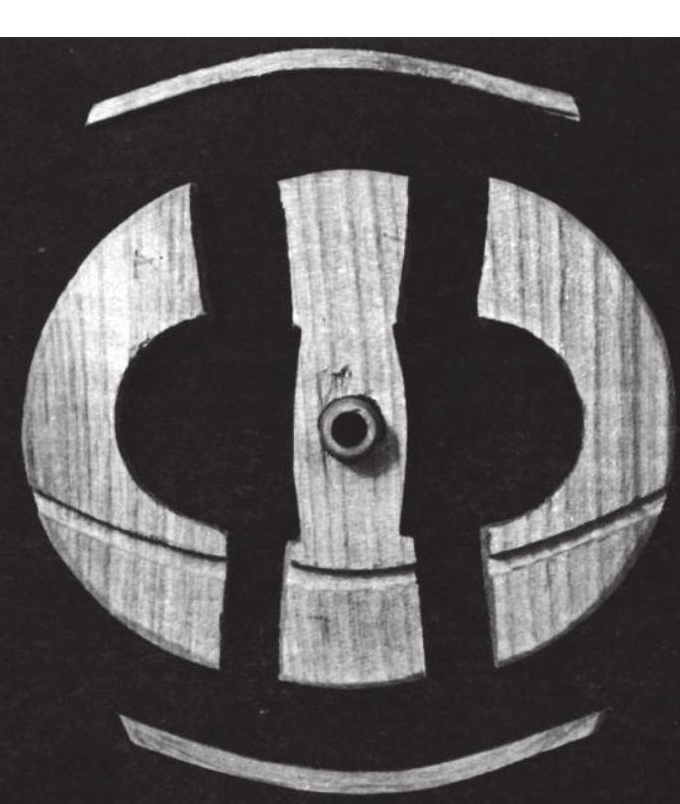


\section{Évolution de l'assemblage à goujons}

L'apparition d'évidements latéraux sur la roue tripartite (fig. 1:2) correspond à l'amélioration identique que l'on observe sur la roue monobloc, et elle vise le même résultat éviter la disjonction des assemblages à la périphérie.

Par la suite, la recherche de l'allégement de la roue amènera l'agrandissement des évidements latéraux, procédé qui laisse apparaître les goujons d'assemblage (fig. $1: 3$ ).

Enfin, cette dernière modification, encore affinée, conduit pendant le $\mathrm{I}^{\mathrm{er}}$ millénaire à un nouveau type de roue (la cross bar wheel des archéologues anglophones) que l'on retrouve à la fois sur les documents figurés antiques (fig. $1: 4$ et 7 ) et dans les découvertes de fouilles (fig. $1: 5$ et 6 ). On trouve encore de nos jours ce modèle de roue aussi bien au Portugal (fig. 1:8) qu'en Mandchourie (fig. 1:9).

\section{Évolution de l'assemblage à éclisses}

1) Sans modification de la structure initiale : la roue tripartite conservera sa forme initiale lorsque les éclisses en bois originelles seront remplacées par des éclisses métalliques et qu'elle sera cerclée d'un bandage en métal permettant de contenir les effets de la dessiccation des bois (fig. $1: B$ et D) ; ce modèle de roue pleine tripartite à éclisses et bandage de fer existe encore au $\mathrm{xx}^{\mathrm{e}}$ siècle (fig. $1: \mathrm{F}$ ).

2) Par transformation de la structure initiale : la solution des évidements latéraux, appliquée à la roue pleine tripartite assemblée par éclisses, implique la suppression de l'éclisse centrale et la courbure des éclisses latérales, qui ménagent la place nécessaire à leur percement (fig. 1 : C). D'autre part, le fait de placer une éclisse sur chaque face de la roue constitue une amélioration, puisqu'en découle une solidité accrue (fig. 7), l'emploi du métal (éclisses de fer et bandage métallique) permettra par la suite d'utiliser ce modèle de roue jusqu'à nos jours, tant dans la péninsule ibérique (fig. $1:$ E et H) qu'au Baloutchistan (fig. $1:$ G).

\section{Observation sur le mode de montage sur l'essieu}

Il ne semble pas possible d'établir une chronologie technique (ou même logique) du mode de montage des roues sur l'essieu. Aux mêmes époques, et des origines jusqu'à nos jours, les roues pleines et leurs dérivés sont, indifféremment, ou bien montées fixes sur l'essieu par une fusée d'essieu en forme de parallélépipède, ou bien montées folles, c'est-à-dire tournant librement sur la fusée, des esses en bout de fusée empêchant simplement les roues de ressortir. La coexistence des deux procédés est encore patente aujourd'hui (voir par exemple les roues E et G, et 8 et 9 de la fig. 1), mais il faut observer que tous ces modèles de roues, pleines et dérivés, ne s'utilisent que sur des véhicules utilitaires campagnards qui ne se déplacent qu'à des allures lentes. Il ne 
peut d'ailleurs en être autrement pour des véhicules à roues fixes dont l'essieu tourne sous la caisse dans des échantignolles, car dans les virages, la roue extérieure, ayant en ce cas plus de chemin à parcourir que l'autre, ne peut que déraper, l'essieu résistant à la torsion, parfois à la limite de la rupture si la charge est importante.

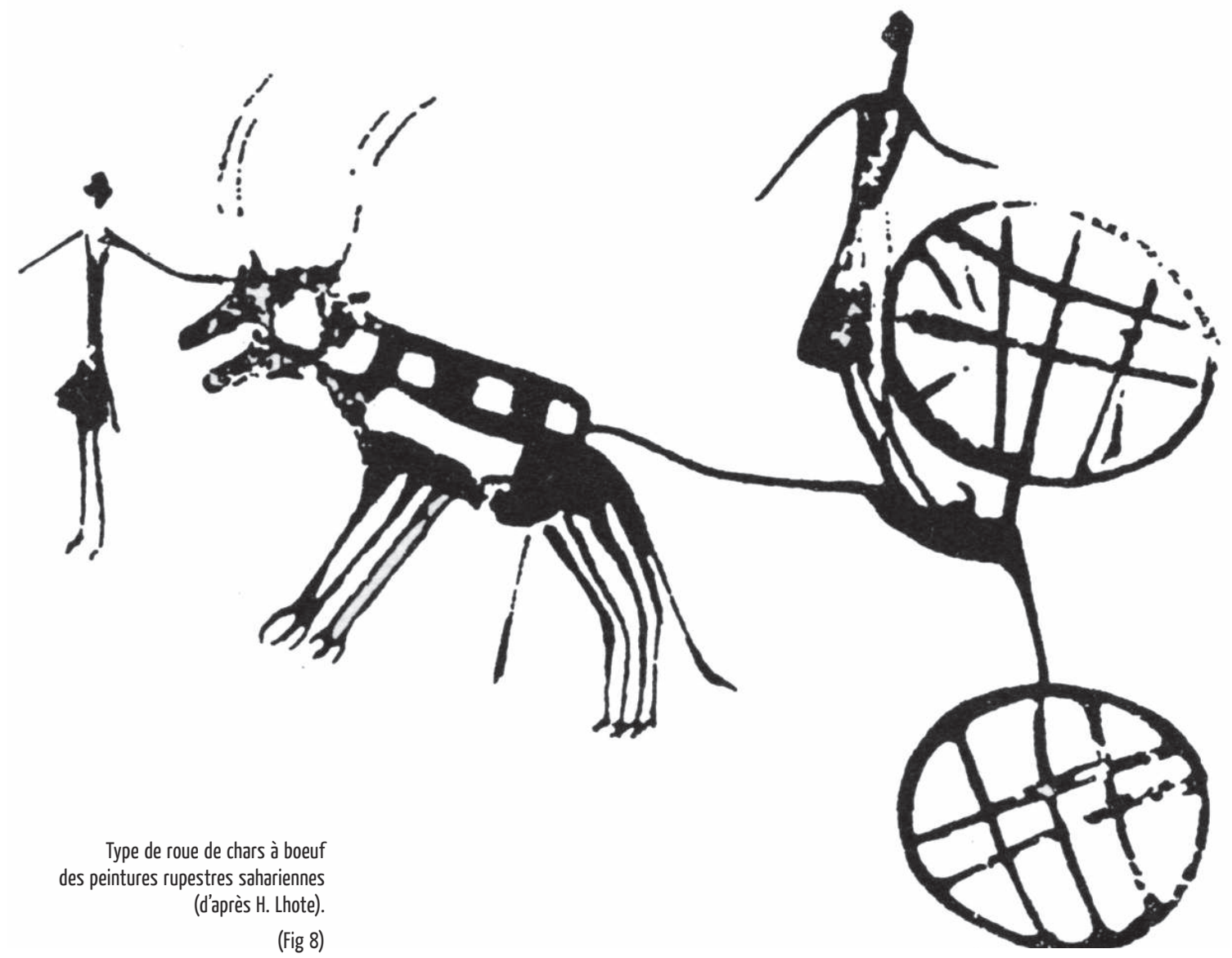


La chronologie technique développée dans cette étude n'est pas en opposition avec la chronologie historique de la roue mise en évidence par la recherche archéologique, mais elle permet cependant de constater que les modèles de roues dérivés de la roue pleine originelle ont suivi une évolution technique différente de celle des roues à rais, avec lesquelles elles ne sauraient être confondues.

D’autre part, l'aire d'extension de la roue pleine et de ses dérivés, touchant toutes les parties de l'Ancien Monde, pose la question de la diffusion de techniques beaucoup trop élaborées pour que l'on puisse penser à de simples convergences. Il semble que la solution de ce problème réside, pour l'essentiel, dans la recherche des moyens matériels d'une telle diffusion; c'est un domaine que l'histoire et l'ethnologie des techniques se doivent d'explorer. 


\section{RÉSUMÉ}

La Roue pleine et ses dérivés. L'auteur expose l'essentiel d'une étude qui s'appuie sur l'analyse des contraintes matérielles qui ont conditionné l'évolution technique de la roue pleine.

Ces contraintes étant invariables à travers l'espace et le temps, la recherche expérimentale sur pièces reconstituées permet d'élaborer la chronologie évolutive des techniques de fabrication des roues depuis l'origine jusqu'à nos jours. En conclusion, ce travail propose une orientation des recherches vers les moyens de communication dans les temps anciens, pour essayer de déterminer comment s'est effectuée la diffusion des techniques beaucoup trop spécialisées pour que l'on puisse envisager de simples convergences technologiques.

\section{ABSTRACT}

The solid wheel and its derivatives. The author sets forth the essential points of a study concerning the physical restraints which have governed the technological evolution of the solid wheel.

These limiting factors being spatially and temporally invariant, experimental research on reconstituted and temporally invariant, experimental research on reconstituted objects leads to the elaboration of an evolutionary chronology of techniques for building wheels from the origins to present times. Finally, this article suggests directing research towards the means of communication in the ancient world to try to determine how were diffused techniques much too specialized to be simply the result of technological convergence.

\section{NOTES ET RÉFÉRENCES DE L'INTRODUCTION}

En ouverture, Roue de char en bois (érable et frêne). Cliché Musée du Laténium, Neuchâtel.

Dégagée lors des fouilles du village de Saint-Blaise/Bain des Dames, à quelques encablures du musée neuchâtelois, cette roue date d'environ 2600 av. J.-C. Les plus anciennes roues conservées ont été mises au jour sur les sites lacustres du pourtour alpin. Réalisées en bois, elles ont été préservées par l'humidité des couches archéologiques lacustres. En plus de celle de Saint-Blaise, on en connaît ainsi plusieurs, qui proviennent notamment des rives du lac de Zurich et du Federsee, dans le sud de l'Allemagne. La plus ancienne roue du monde (env. 3200 av. J.-C.) est une découverte très récente : elle a été mise au jour dans un village palafittique des marais de Slovénie, sur le site de Stare Gmajne.

Smil, V. 2000 Horse power, Nature $405: 125$.

Sigaut, F. 2000 Ancestor knews how harness horsepower, Nature 408 : 287-288. 